\title{
Key questions in cardiac surgery
}

\author{
N Moorjani. N Viola. SK Ohri
}

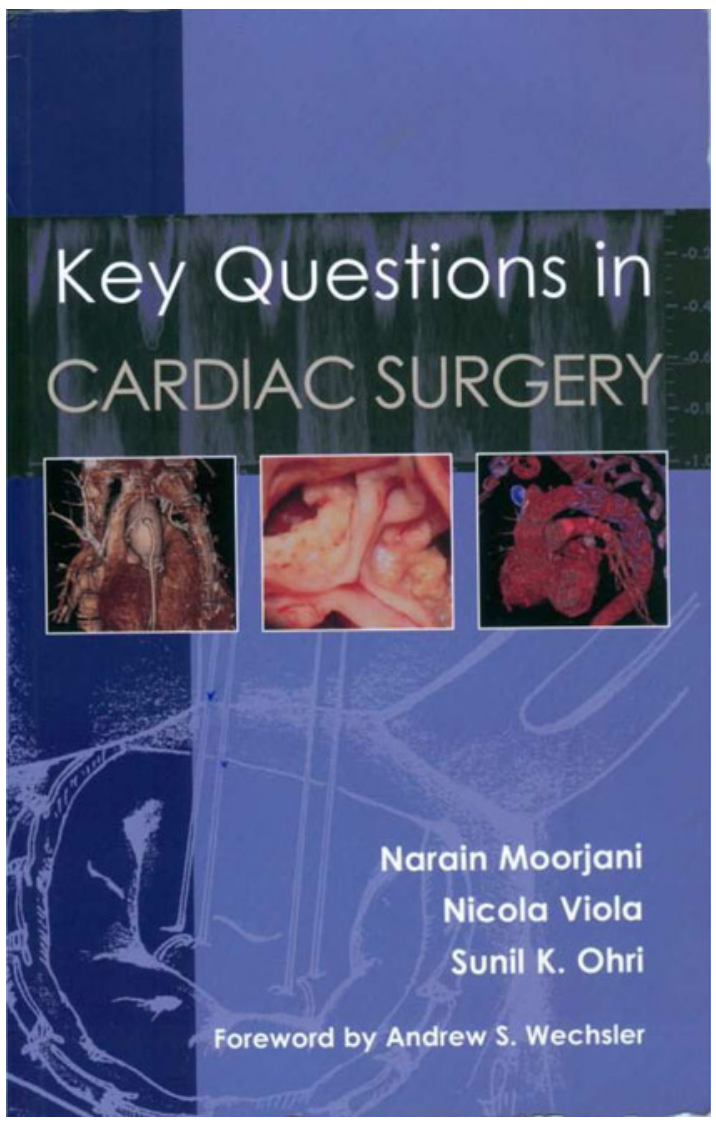

\footnotetext{
A. S. Kumar $(\bowtie) \cdot$ A. K. Sharma

Cardiac Surgery, Pushpanjali Crosslay Hospital,

Ghaziabad (UP), India

e-mail: ijtcvs2000@hotmail.com
}

The title of this book "Key Questions in Cardiac Surgery" is appropriate since it covers systematically all questions asked in examinations or during ward round. This book covers all acquired adult cardiac diseases. This book also covers related embryology, anatomy and physiological basis for these acquired lesions. It is written in a questionanswer format which makes it interesting to read.

The illustrations are very good. Diagrams have been made in such a way that they provide a surgeon's view. Color scheme used in diagrams are relevant and makes the description easy to understand. For example the diagram depicting complications of Arterial cannulation is eye catching that it gets imprinted into mind of reader. Photographs are truly representative of respective pathologies.

This book based on the guidelines issued by American Heart Association and European Society of Cardiology, and incorporates facts based on current scientific literature. Each chapter has a list of key scientific references for further reading.

This book covers extensively Cardiac Anatomy, Physiology, Pharmacology, Electrocardiography, ECHO cardiogarphy, Cardiac Catheterisation, Radiological investigations and Cardio-pulmonary Bypass. These chapters are very important a good understanding of cardiac disease and the surgical procedures. For example understanding the basis of Positron Emission Tomogram scan works, gives scientific basis to a trainee surgeon when he orders this for his patient!

The subject index is very good and makes relevant information easy to access.

We would recommend this book as a companion for all Cardiac Surgery and Cardiology Trainees, Perfusionist and Cardiac Anesthesiologist.

Over all, this book provides a practical and new approach to understand Adult cardiac surgery. The book does not cover congenital heart surgery, Thoracic surgery or surgery for peripheral vascular diseases.

\section{A. Sampath Kumar • A. K. Sharma}

